\title{
Dynamics of immunological indices in the exhaled air condensate in patients with bronchial asthma on the background of pathogenetic therapy with the use of I-arginine
}

\begin{abstract}
The aim of the study was to study the effect of L-arginine on the content of certain interleukins in the exhaled air condensate (CVC) in patients with bronchial asthma. 52 patients with a persistent asthma of a moderate course were examined. Two groups were formed. Patients of the main group (26), in addition to the standard basic therapy, received a nitric oxide donor - L-arginine. The comparison group (26) received only basic therapy. In patients of both groups before and after treatment, certain parameters of the immune system (IL-4, TNF- $\alpha$ ) were measured in CMV. The initial data in patients of both groups were characterized by an increase in IL- 4 and TNF- $\alpha$ in KVV compared with the group of healthy individuals. The content of IL-4 and TNF- $\alpha$ in KVB against the background of L-arginine significantly decreased during the control period. Changes in these parameters in the control group were not so pronounced and were unreliable. The inclusion of L-arginine in the baseline therapy contributed to an earlier and significant decrease in the content of the studied interleukins.
\end{abstract}

Keywords: bronchial asthma, 1-arginine, interleukins, endothelial dysfunction, exhaled air condensate
Volume 5 Issue I - 2018

\author{
Alyavi AL, Bazarova SA, Dzhambekova GS, \\ Kasymova GM \\ Republican Specialized Scientific and Practical Medical Center \\ for Therapy and Medical Rehabilitation Tashkent, Uzbekistan
}

\section{Correspondence: SA Bazarova, Republican Specialized Scientific and Practical Medical Center for Therapy and Medical} Rehabilitation Tashkent, Uzbekistan, Email d-sayyorik@mail.ru

Received: December 27, 2017 | Published: January 24, 2018

\section{Introduction}

Bronchial asthma is a severe chronic respiratory disease and represents a major public health problem. ${ }^{1}$ At present, cardiovascular effects are considered as systemic manifestations of asthma, among which endothelial dysfunction appears as the primary link of the vessel wall damage. ${ }^{2}$ Dysfunction of the endothelium, showing up already in the early stages of the disease, aggravates the increasing respiratory failure, hypoxemia and tissue hypoxia. ${ }^{3}$ This contributes to the emergence of severe violations of systemic and pulmonary hemodynamics. In recent years, new data have been obtained on the role of disturbances in the functional activity of the endothelium in the pathogenesis of bronchopulmonary diseases. ${ }^{4}$ Objective registration of the vascular endothelium status is important not only for assessing the degree of its dysfunction, the selection of pathogenetically justified drug and non-drug treatment, but also for determining the prognosis of the course of the disease. ${ }^{5}$ Despite the emergence of new classes of drugs for the treatment of asthma, a significant number of patients with severe forms of this disease remain, who need a long-term intake of a number of drugs, which, as it turned out, have a damaging effect on the vascular wall. ${ }^{6}$ In recent years, with lung diseases,,${ }^{6,7}$ the role of nitric oxide is actively studied, which is one of the most important mediators of the functioning of various organs and systems. ${ }^{3,8}$ And in the treatment tactics much attention is paid to amino acids, which have a therapeutic effect. Arginine is a conditionally indispensable amino acid, but its deficiency leads to the rapid development of pathological processes. Under physiological conditions, $\mathrm{NO}$ is synthesized from L-arginine by means of NO synthase-NO (NOsynthase) enzymes. ${ }^{8}$ Many of the effects of arginine are due to the fact that it is the pre-agent of $\mathrm{NO}$ (donor) produced by endothelial cells of the vessels, macrophages and neutrophils. ${ }^{8} \mathrm{BA}$ as a complex chronic inflammatory disease involves many structural cells and inflammatory cells, followed by the release of inflammatory mediators, which lead to the formation of the main pathophysiological mechanisms of the disease. ${ }^{1,6}$ At present, about 50 cytokines and chemokines are known to play an important role in the pathogenesis of asthma, ${ }^{7}$ but their role in the management of pathophysiological processes has not been fully understood. Investigation of markers of inflammation in the lungs is usually carried out by taking material using bronchoalveolar lavage (BAL), bronchobiopsy, ${ }^{8}$ as well as serum and urine to assess systemic effects. However, the production of BALF and bronchobiocytes are invasive, traumatic methods that cause multiple side effects, which cannot be carried out in a short time, as well as in severe patients, children and in outpatient practice. ${ }^{1,2}$ In recentyears, non-invasive methods for diagnosis and control of asthma treatment have been widely introduced into clinical practice, in particular, the study of biomarkers in the exhaled air condensate. ${ }^{5}$ Analysis of CVI allows to evaluate the activity of inflammation in the lungs and to clarify the state of local inflammation in the airways, as well as assess the role of cytokines in it.

\section{Materials and methods}

We examined 52 patients aged 18 to 55 years ( $38.5 \pm 4.2$ years) with persistent asthma of moderate course, with duration of disease on average $15 \pm 2.3$ years, and 15 healthy volunteers. By the method of random sampling, two groups comparable in age and sex were formed. Patients of the main group $(\mathrm{n}=26)$, in addition to standard basic therapy (GINA, 2007) received a nitric oxide donor - L-arginine. The drug (100 $\mathrm{ml}$ of a $4.2 \%$ solution, Tivortin, YURIYAFARM, Ukraine) was injected iv by drop, once a day, for 10days. The comparison group $(n=26)$ received only basic therapy. In patients of both groups, the parameters of the immune system were evaluated before and after treatment. In IVC, the content of interleukin-4 (IL-4), tumor 
necrosis factor- $\alpha$ (TNF- $\alpha)$ was determined. The collection of KVV was carried out by the method of GI Sidorenko et al. (1980) modified by us. The determination of cytokines (IL-4, TNF- $\alpha$ ) in CMC was performed by the method of enzyme immunoassay. Reagent kits for immunoenzymatic analysis of human cytokines from VectorBest (Novosibirsk) were used. The received data are processed on a personal computer in the Microsoft Excel software environment using the built-in "Analysis package", which is specially designed for solving statistical problems.

The initial data in patients of both groups were characterized by an increase in the content of IL- 4 and TNF- $\Phi \alpha$ in CMV compared to the group of healthy individuals. The effectiveness of treatment with the inclusion of L-arginine was manifested by an improvement in clinical symptoms. The content of IL-4 and TNF- $\alpha$ in KVB against L-arginine to the control period (10days from the beginning of therapy) significantly decreased $(p<0.05)$. Changes in these parameters in the control group were not so pronounced and were unreliable. The inclusion of L-arginine in the baseline therapy was accompanied by a significant decrease in the content of the studied interleukins (Table $1)$.

Table I Dynamics of immunological indicators in CVI on the background of treatment

\begin{tabular}{|c|c|c|c|c|c|c|}
\hline \multirow{2}{*}{ № } & \multirow{2}{*}{ Indicators } & \multicolumn{2}{|c|}{ Main group $(n=26)$} & \multicolumn{2}{|c|}{ Control group $(n=26)$} & \multirow[t]{2}{*}{$\begin{array}{l}\text { A group of healthy } \\
\text { individuals }(n=\mid 5)\end{array}$} \\
\hline & & To & After & To & After & \\
\hline I & IL-4 (pg/ml) & $1,49 \pm 0,53$ & $0,94 \pm 0,50 *$ & $|, 47 \pm 0,5|$ & $1,39 \pm 1,3 * * *$ & $0,8 I \pm 0,36$ \\
\hline 2 & TNF- $\alpha(\mathrm{pg} / \mathrm{ml})$ & $4,35 \pm 2,14$ & $3,86 \pm 1,18 *$ & $4,38 \pm 1,9$ & $4,02 \pm 1,9 * * *$ & $3,80 \pm 1,45$ \\
\hline
\end{tabular}

*,Value level of the initial data $\mathrm{p}<0.05$

**, Level of reliability of data between groups $p<0.01$

\section{Conclusion}

Thus, the conducted study showed that, in patients with a long course of persistent asthma of moderate severity, in addition to the basic therapy of the donor nitrogen oxide (Tivortin), a number of immunological indices are improved. Received preliminary positive results dictate the need for further study of the mechanisms of L-arginine influence on the endothelial function in bronchial asthma and the possibility of its preventive use.

\section{Acknowledgements}

None.

\section{Conflict of interest}

Author declares that there is no conflict of interest.

\section{References}

1. Chuchalin AG. Bronchial asthma M: Russian physician. 2001.

2. Vanin AF. Nitric oxide in biomedical research. Vestn Ross Akad Med Nauk. 200;4:3-5.
3. Gozhenko AI, Kotyuzhinskaya SG, Kotyuzhinsky AI. Role of nitric oxide in the regulation of microcirculation and the aggregate state of blood. Ukr med almanac. 2000;(1):13-17.

4. Danilova EI, Grafova VN, Kukushkin ML, et al. Effects of L-arginine in the cerebral-spinal pain syndrome Bul experiment biol and honey. 1999;(2):160-163.

5. Zefirov AL, Haliullina RR, Anuchin AA, et al. Influence of endogenous nitric oxide on the function of the neuromuscular synapse ROS fiziol. IZM Sechenova. 2001;(4):S499-506.

6. Barbul A, Iazarou SA, Efron DT, et al. Arginine enhances wound healing and lymphocyte immune responses in humans. Surgery. 1994;108(2):331-337.

7. Beaux de AC, O'Riordain MG, Ross JA, et al. Glutamine-supplemented total parenteral nutrition reduces blood mononuclear cell interleukin-8 release in severe acute pancreatitis. Nutrition. 1998;14(3):261-265.

8. Busse R. Fleming I Regulation and functional consequences of endothelial nitric oxide formation. Ann Med. 1995;27(3):331-340. 